\title{
Les réseaux sociaux et l'échange entre l'homme politique et les internautes : le cas de Facebook après les élections présidentielles en France
}

Social Networks and the Exchange Between Politicians and Surfers: Facebook and the presidential post elections in France

\section{Galia Yanoshevsky}

\section{(2) OpenEdition Journals}

\section{Édition électronique}

URL : http://journals.openedition.org/aad/1008

DOI : 10.4000/aad. 1008

ISSN : 1565-8961

\section{Éditeur}

Université de Tel-Aviv

\section{Référence électronique}

Galia Yanoshevsky, "Les réseaux sociaux et l'échange entre l'homme politique et les internautes : le cas de Facebook après les élections présidentielles en France ", Argumentation et Analyse du Discours [En ligne], 5 | 2010, mis en ligne le 20 octobre 2010, consulté le 23 septembre 2019. URL : http:// journals.openedition.org/aad/1008; DOI : 10.4000/aad.1008

Ce document a été généré automatiquement le 23 septembre 2019.

\section{(c) $(1) \subseteq$}

Argumentation \& analyse du discours est mis à disposition selon les termes de la licence Creative Commons Attribution - Pas d'Utilisation Commerciale - Pas de Modification 4.0 International. 


\title{
Les réseaux sociaux et l'échange entre l'homme politique et les internautes : le cas de Facebook après les élections présidentielles en France
}

\author{
Social Networks and the Exchange Between Politicians and Surfers: Facebook \\ and the presidential post elections in France
}

Galia Yanoshevsky

1 Facebook fait partie d'un nombre croissant de réseaux sociaux sur Internet qui s'emploient à favoriser l'échange entre gouvernants et gouvernés. Il permet d'entériner l'idée, exprimée dès 2000, selon laquelle l'Internet contrarie les efforts accomplis au cours des trente dernières années par les grands partis pour centraliser leur fonctionnement ainsi que pour unifier et professionnaliser leur communication (Vedel $2000: 28)$.

2 C'est en ce sens que Vedel (ibid.: 30) prédisait que «les réseaux électroniques permettraient de transformer les principes et les mécanismes de la représentation politique dans le sens d'une démocratie directe ". À une vision optimiste, qui voit dans l'interactivité du Web 2.0 (Vedel 2000, 2003; Vaccari 2008; Greffet \& Wojcik 2008; Flichy 2008) un outil technologique conduisant à une réalisation de l'idéal de la sphère publique à la Habermas, et résultant en une décentralisation de la politique et en une démocratie plus directe, sans médiation entre l'homme politique et les citoyens, s'oppose cependant une vision pessimiste. Selon elle, les TIC (technologies de l'information et de la communication) favoriseraient non pas la subjectivation autonomisante, mais bien l'assujettissement, car elles n'empêchent pas les processus liés aux rapports de pouvoir (Goupil 2007; Greffet \& Wojcik 2008 ; Flichy 2008). Sans opter a priori pour l'une de ces deux positions, on tentera ici d'explorer sur le terrain les 
modalités d'interaction entre l'homme politique et les internautes sur Facebook afin de mieux en saisir la logique et les enjeux.

3 Le but de cet article consiste donc à analyser les fonctionnements des réseaux sociaux à travers l'exemple de Facebook - sur le Web 2.0 dans les interactions de l'homme politique avec ses soutiens après la campagne présidentielle de 2007 en France. Il s'agit de voir comment ce dispositif, qui a priori n'est pas un outil politique hiérarchisant, ni d'ailleurs un forum consacré aux discours politiques, est exploité par diverses personnalités pour leurs besoins propres (construction d'image, rassemblement, information), et comment l'internaute peut le récupérer à son profit par diverses stratégies. Au niveau pratique, l'homme politique a recours à toutes les stratégies identifiées par les études des TIC, à savoir la conversationalisation ${ }^{1}$ et le simulacre de la proximité et de l'intimité, procédures qui sont susceptibles de rapprocher les soutiens. Les internautes, quant à eux, se montrent à la fois crédules et ouverts aux initiatives des hommes politiques, mais aussi prudents et vigilants. Diverses stratégies d'appropriation leur permettent alors de regagner du terrain sur leur espace de libre discussion. A travers cette analyse, on essayera de voir dans quelle mesure Facebook sert aujourd'hui d'agora, et quelles en sont les avantages et les insuffisances.

\section{Facebook : Histoire et caractéristiques}

4 Facebook fait partie des dispositifs de réseaux sociaux comme MySpace, YouTube, Flickr, etc. offerts par Internet. Un réseau social est un ensemble de personnes, organisations et autres entités sociales liées par un ensemble de relations sociales significatives (Williams \& Gulati 2007: 3). Un réseau informatique devient social lorsqu'il lie des personnes (Wellman 1997, cité ibid.). Ces réseaux ont des objectifs très différents de ceux des autres sites sur la toile. Des sites comme MySpace et Facebook favorisent en effet l'émergence de nouvelles relations entre les individus (Obersdorff 2009 : 13) et deviennent de ce fait de plus en plus populaires auprès des politiciens et de leurs organisations comme moyen de diffuser des messages politiques, de découvrir les domaines d'intérêt et les besoins des électeurs et de la population en général, de recueillir des fonds et de constituer des réseaux de soutien. Le contenu affiché sur ces sites peut se rapporter à des questions politiques et au travail officiel des politiciens, mais aussi à des aspects de leur vie personnelle (Clark $2010: 1$ ).

5 Ils servent à informer le citoyen et l'interpeller (Clark $2010: 4$ ). Mais, si les hommes politiques peuvent en principe contrôler le contenu de leurs sites officiels, les sites des réseaux sociaux proposent au contraire aux internautes des espaces de libre échange, sans censure de la part des représentants politiques. Ils leur donnent aussi l'occasion d'initier un contact avec d'autres usagers (Williams \& Gulati $2007: 4$ ). En effet, tandis que dans le passé, le seul feedback offert aux candidats par les électeurs provenait des sondages, à partir de 2004, Internet a permis d'envisager d'autres moyens de connaître l'intérêt manifesté par le public et de connaître ses opinions, facilitant ainsi l'engagement des citoyens dans le processus politique (Westling 2007).

6 A en croire Gueorguieva (2008: 288), les sites de réseaux sociaux se sont rapidement transformés en phénomène de masse. En 2007, déjà plus de trois millions de Français les utilisent (Etudes et Plas, 14 décembre 2007, cité chez Obersdorff 2009 : 13). En 2008, 59 \% des Canadiens ont créé un profil sur un réseau social (Small $2008: 85$ ). Aux EtatsUnis, une partie importante des électeurs se sert de réseaux sociaux comme YouTube et 
MySpace, ce qui rend pertinente leur inclusion dans les stratégies de campagnes électorales (ibid.). C'est le cas aussi de Facebook, plateforme généraliste fondée en février 2004 et qui s'annonce comme un outil social liant les gens avec des amis et d'autres personnes qui travaillent, étudient et vivent dans leur entourage ${ }^{2}$. Il attire aujourd'hui près de 140 millions d'inscrits dans le monde (Cardon 2009: 11), et figure parmi les outils qui permettent aux campagnes électorales d'organiser et de communiquer avec les individus qui soutiennent le candidat de manière efficace (Westling $2007: 2$ ). Tandis que Friendster et MySpace sont issus des réseaux sociaux, Facebook constitue aujourd'hui l'un des sites Web les plus visités (Small $2008: 85$ ) ${ }^{3}$.

7 Le grand succès de Facebook en tant qu'outil de promotion politique s'est confirmé avec Barack Obama, qui l'a emporté sur McCain. Le site du président à l'Elysée comporte aujourd'hui le statut marital, les activités sportives et les goûts littéraires du président (site officiel sur Facebook de Sarkozy). Facebook devient donc un outil essentiel des hommes et des candidats politiques, avec cependant des degrés changeants d'interactivité, comme nous allons le constater par la suite ${ }^{4}$.

\section{Corpus et méthodologie}

8 Mon corpus consiste en un ensemble représentatif d'échanges sur Facebook dans les sites officiels des personnalités politiques. Suite à une étude (Yanoshevsky 2009) sur le fonctionnement de leurs sites électoraux officiels, je me limiterai, dans le contexte français, aux deux candidats principaux de la dernière campagne électorale (2007) : Nicolas Sarkozy et Ségolène Royal, dont le rythme cybernétique est considérablement différent pendant et après les élections ${ }^{5}$.

9 La période examinée est celle de l'après-élection, et plus spécifiquement au lendemain du deuxième tour (7 mai 2007 et jusqu'en juin 2010). Il s'agissait de suivre les sites des deux personnalités au quotidien afin de relever des exemples des posts respectifs de Sarkozy et de Royal, d'une part, et des internautes, d'autre part. Les exemples sont choisis en fonction de leur représentativité, à savoir, leur fréquence, mais aussi en fonction de leur singularité (des messages irréguliers qui éclairent le fonctionnement «normé » de l'usage politique de Facebook). Ma démarche consiste à étudier les posts, donc les modes d'échange en temps différé entre les hommes politiques et les internautes sur les pages officielles. En me situant dans le cadre de l'analyse du discours de la CMO (communication médiatisée par ordinateur), je me propose de décortiquer les messages individuels dans leurs environnements respectifs : selon les différents «tabs" (catégories préétablies ou catégories proposées sur le site de la personne politique) et selon les différents thèmes et moments de réaction. Pour des raisons techniques et pour la clarté de l'analyse, je présente d'abord les posts des hommes politiques, puis je procède à l'examen des réactions des internautes.

Les messages des personnes politiques et de leurs soutiens montrent que Facebook accueille des échanges virtuels de tout ordre : messages postés sur des "murs », sprtes de tableaux virtuels où chacun peut afficher des messages (visibles pour les adhérents ou pour tout le monde, au choix), une partie «info » où l'on peut rajouter des détails personnels, des sections «notes", "boxes ", " photos", «vidéos ». Etant donné qu'il s'agit d'un dispositif extrêmement facile à manipuler et hautement modulaire, chacun peut choisir les catégories et les employer à son gré. 


\section{Les hommes politiques sur Facebook : vedettisation et information}

11 Facebook privilégie à la fois une présentation individualisée de soi (statut personnel, passe-temps, goûts, photos, etc.) et une manifestation de ses relations sociales. Ainsi, lorsque l'homme politique se présente sur Facebook, il est à la fois individu (Vanbremeersch 2009) et représentant. Sa présence sur ce réseau social est en même temps une confirmation des règles du jeu de Facebook qui consistent en une présentation de soi par une série d'images et de posts afin de se connecter avec d'autres «amis » ou individus sur le réseau, et en un acte de marketing politique destiné à gagner plus d'adhérents ou à mobiliser les soutiens existants. En effet, comme d'autres dispositifs publics sur Internet, Facebook se prête facilement au jeu du marketing politique, et les hommes politiques s'en emparent. Existant à la fois nulle part et partout, les échanges sur Facebook remplacent les déplacements (Le Bart 2007 : 43) et les poignées de mains, pratiques qui précédaient l'âge télévisuel et correspondent aux adresses personnelles des candidats à la télévision (Bonnafous et al. 1999). En même temps, la latitude de son format permet la diffusion de messages informationnels de taille changeante.

12 En parcourant les messages postés par Nicolas Sarkozy et par Ségolène Royal sur leurs sites officiels respectifs de Facebook, on constate que deux tendances majeures y règnent : d'une part, une forte disposition à construire une image de soi qui joue sur la minimisation de l'effet de surplomb et parallèlement - l'augmentation de la proximité (selon les termes de Le Bart 2007). D'autre part, on trouve une tentative d'informer les internautes/soutiens de l'action menée par l'élu/ le représentant politique. Ces deux tendances, présentes chez les deux anciens candidats aux présidentielles, se manifestent pourtant de façon assez différente chez le Président de la République et chez la représentante de la région Poitou-Charentes.

13 Alors qu'une grande partie des messages sur le site officiel de Sarkozy sont formels, ayant pour formule « Monsieur le président » ou «Le Président de la République » (des messages qui rapportent ses activités ou ses opinions sur un sujet donné), les messages personnels, lorsqu'ils apparaissent, ont recours à un autre ton. Par exemple, Sarkozy traite explicitement de ses problèmes de santé en juillet 2008, et remercie les internautes à la première personne :

[Nicolas Sarkozy] remercie tous ses supporters sur Facebook pour leurs messages de soutien.

Tuesday, July 28, 2009 at $10: 08 \mathrm{pm}$

Je vais très bien après le petit incident de dimanche, qui m'incite à prendre du repos cet été.

Merci pour vos encouragements et témoignages, qui me touchent beaucoup.

Parmi tous les messages que j'ai pu recevoir - de tous mes collègues chefs d'Etat et de gouvernement étrangers notamment - les vôtres me tiennent particulièrement à cœur.

Votre sympathie et votre spontanéité sur Facebook font vivre un lien précieux entre nous Je vous souhaite à tous un très bon été. Nicolas Sarkozy

Le registre est personnel, et singularise les voeux des soutiens en les mettant au-dessus des vœux officiels. La répétition d'un vocabulaire émotif et expressif («me touchent 
beaucoup ", "les vôtres me tiennent particulièrement à cœur ", "votre sympathie ", "font vivre un lien précieux entre nous ») semble minimiser l'écart entre l'élu et l'homme de la rue en suggérant par inférence que, comme tout autre être humain, le Président de la République est fragile et sensible. Ainsi, une illusion d'intimité se produit, où le président semble être quelqu'un de qui on peut se sentir proche. Il peut être considéré par son auditoire comme un ami, un conseiller, quelqu'un qui réconforte (et est réconforté) et un modèle (Horton \& Whol 1956 : 216-217).

Quoique partageant le même style personnalisé, le message suivant a un objectif tout autre : c'est le message introductif du site de Sarkozy, qui l'initie au jeu de Facebook:

Chers amis, Merci à vous de m'avoir rejoint sur cette page créée par mon équipe pour mieux répondre à votre demande d'information et de transparence. N'hésitez pas à me dire ce que vous aimeriez y trouver. Le temps me manque mais soyez persuadé que je me tiens informé de vos échanges et que j'apprécie les témoignages de sympathie que vous m'envoyez. Faisons vivre ce lien entre nous. Nicolas Sarkozy May 22 at $05: 35 \mathrm{pm}$

Visiblement, il s'agit de voir en Facebook un canal de transmission d'information entre l'élu et le peuple ( «pour mieux répondre à votre demande d'information et de transparence »). Mais l'essentiel de ce message réside dans la manière dont se construit l'image du candidat: c'est quelqu'un qui manifeste de la bonne volonté à l'égard de ses soutiens (" n'hésitez pas à me dire ce que vous aimeriez y trouver »), mais qui est aussi franc en avouant le fait que c'est son équipe qui a érigé le site pour lui, et qu'il se « tient informé» (plutôt que de participer activement). Se dessine donc l'image d'une personne honnête qui reconnaît les limites de son travail.

En effet, Sarkozy participe au jeu de posting sur sa page officielle de Facebook, mais ces messages (au moins dans la première période analysée) ont tendance à être personnels, relevant des affaires quotidiennes ou familiales à l'Elysée. Ainsi, l'internaute qui découvre la page du président s'informe des activités culturelles de celui-ci, à savoir, le film Home du réalisateur Yann Arthus-Bertrand qu'il a vu récemment (Sarkozy va jusqu'à en faire un commentaire, cf. message du 4 juin 2009), la rencontre avec Woody Allen et les Gates (message de 27 mai 2009), et ainsi de suite. Le brouillage entre le personnel et le public est renforcé davantage encore par des vidéos de l'épouse du président qui est interviewée par un magazine féminin (FemmesActuelles; message du mai 18 2009). Le président y apparaît en pleine interview et se montre affectueux à l'égard de sa femme et détendu vis-à-vis des interviewers. Les gestes familiers (s'installer dans un fauteuil, à côté de sa femme, l'embrasser sur la bouche,...), se situent dans le prolongement de la ligne déjà manifestée dans les séquences vidéo sur le site officiel du candidat aux présidentielles (cf. Yanoshevsky 2009), où il produit une image décontractée de lui-même.

18 Dans le cadre de tels posts, on voit s'entremêler des aspects/codes de la sphère publique et privée. Selon la distinction du monde antique, la sphère privée concerne tout ce qui a rapporte à la maison ou à la famille, tandis que le terme de sphère publique est réservé à la polis (Poster 1997 : 265). Qui plus est, dans le langage ordinaire, le terme "privé " est utilisé pour désigner tout ce qui n'est pas exposé aux yeux du public : des discours et des actions isolés, que personne ne remarque et qu'on n'enregistre pas (ibid. : 265). La sphère publique désigne au contraire l'espace où les citoyens discutent de leurs affaires communes. Les candidats aujourd'hui «viennent plutôt mettre en valeur leur personnalité à travers leur vie privée comme leur vie publique [...] Dans un monde où parole privée et parole publique se mélangent de plus en plus, il n'est pas étonnant 
qu'Internet propose à ses utilisateurs d'interconnecter leurs discours " (Flichy 2008 : 169).

Répondant sans doute à l'intérêt manifesté par le public, Sarkozy montre sa vie privée lorsqu'il poste sur son site des vidéos et des images avec sa célèbre épouse (des « scènes de la vie privée »). Par là, il donne a voir " une image concrète de la vie de tous les jours » (Cong Huyen Nu 1995 : 55), à l'instar des images qu'on avait déjà connues à l'ère de la télévision. Il obéit par ailleurs aux processus de vedettisation des élus dans les années 2000 (Dakhlia 2008: 66), se construisant en même temps une image d'homme ouvert qui répond aux désirs voyeuristes d'un auditoire qui veut prendre connaissance de la vie du couple célèbre. ${ }^{6}$ Enfin, il semble obéir à une autre règle des célébrités (les people) qui, selon Tolson (2001) serait le va-et-vient constant entre ce que Tolson apelle le caractère extraordinaire et le caractère ordinaire des gens célèbres dans leur image publique.

C'est tout le contraire chez Ségolène Royal qui établit une séparation nette entre sa vie publique et privée, ce qui apparait clairement dans le choix des photos et des vidéos sur sa page officielle de Facebook : toutes manifestent son être public, aucune ne dévoile ses activités familiales.

21 Il semble qu'ici Facebook ne fasse que renforcer des tendances qui sont présentes ailleurs et avant lui. La différence entre ces deux personnalités politiques relève sans doute en partie de leur statut respectif (on s'intéresse plus à la vie privée du Président de la République qu'à celle d'une présidente de région), soit de leur statut marital différent : alors que la vidéo de l'heureux couple Sarkozy-Bruni peut être considérée comme une variation de la photo de la famille présidentielle heureuse, telle que l'avait décrite Barthes dans Mythologies (1957), la vie privée de Royal est loin de correspondre aux stéréotypes de la famille heureuse. L'usage de Facebook ne fait donc que renforcer la tendance des anciens médias à exposer (ou au contraire, voiler) des fragments de la vie privée des politiciens en faveur de la construction d'une image qui correspondrait aux attentes stéréotypées du rôle public qu'ils jouent.

Les modalités selon lesquelles Ségolène Royal construit une image positive de sa personne - à savoir une représentante politique qui fait son travail - passe donc moins par l'exposition de sa vie privée sur le Web, que par des appels à agir ensemble. Ainsi, sur son «mur», on trouve des messages qui annoncent ses activités publiques et sa politique ainsi que des appels à la participation/ mobilisation (message du 11 août 2009: «Face à une sécheresse qui s'accélère, Ségolène Royal, Présidente de la Région Poitou-Charentes, demande une accélération des décisions...»; message du 19 mai 2010 : « Les attaques de la Droite contre les propositions du PS : trop violentes pour être honnêtes. Prenez la parole sur le site Désirs d'Avenir, participez, prenez votre avenir en main !»). Par ailleurs, les lettres ouvertes, adressées aux dirigeants ou aux internautes, constituent un autre moyen d'action politique. Dans une mise en scène de la transparence, mais aussi de monstration politique, elle utilise le dispositif afin de communiquer des lettres ouvertes écrites en style formel à d'autres hommes politiques, comme dans l'exemple suivant :

Ligne LGV Sud-Europe Atlantique : Lettre au Premier Ministre

Poitiers le 31 juillet 2009 Monsieur le Premier Ministre, Dans le cadre du financement de la ligne LGV Sud-Europe Atlantique pour laquelle le Gouvernement exige une participation des collectivités territoriales, je vous ai proposé le 19 juillet dernier une solution recueillant le consensus de [...] 
Au-delà de l'usage de la lettre ouverte, genre typique de la presse écrite où il a souvent pour objet de constituer ipso facto un groupe virtuel de soutien ou de complicité à l'encontre du destinataire, cette démarche donne à voir une certaine image d'elle qu'elle cultive aussi ailleurs, celle de protectrice des intérêts régionaux. Car, comme de règle dans le genre de la lettre ouverte, celle-ci comporte une adresse directe (ici, au premier ministre) mais est en fait destinée aux tiers - les overhearers - ou sympathisants de Ségolène Royal qui lisent cette page. Dans une autre lettre ouverte (message du Juillet 24, 2009), adressée cette fois-ci directement aux sympathisants et signée de sa plume, Ségolène Royal propose dans son style participatif particulier une critique du Parti Socialiste, un remerciement à ceux qui l'ont soutenue et des invitations à des activités régionales et civiles. Tandis que la signature fac-similée (d'une écriture lisible, personnelle comme dans les mailings dits " personnalisés », sans rapport avec le paraphe d'un ministre signant des centaines de documents) offre une touche personnelle en dépit des contenus formels de la lettre, les thèmes abordés et le style qui les véhicule préservent en fait l'image participative offerte par Ségolène Royal: on le voit dans les formules d'adresse et les personnes grammaticales («L'été nous invite à faire une pause, à prendre du recul et de la hauteur "; "Avec vous, j'ai porté les couleurs de la gauche à l'élection présidentielle de 2007. Avec vous, j'ai porté l'exigence de la rénovation lors du débat du Congrès »; ibid., je souligne). On le voit aussi dans les déclarations concernant la volonté d'unité dans son parti politique et l'invitation à participer à la fête de la fraternité.

Dès lors, lorsque Ségolène Royal emploie des procédés personnels et s'adresse directement aux internautes, elle n'entend pas produire une image positive de soi à travers une manifestation de bonne volonté, comme le fait Sarkozy: il s'agit au contraire de construire l'image positive d'une personne qui désire la participation citoyenne. Par exemple, le marqueur «discussion» (absent chez Sarkozy, et en fait, custom-made chez Royal), exhibe une longue liste de discussions politiques, sans la moindre intervention de la représentante, où de véritables arguments et contrearguments se donnent à lire (J'y reviendrai dans l'analyse des réactions des internautes).

\section{Les cybercitoyens - réoccuper le terrain}

Le problème du libre usage que font les internautes des sites publics est abordé dans la littérature sur les discours politiques en ligne comme un de ses véritables enjeux. De nombreux chercheurs l'interprètent comme une menace à l'égard des personnalités politiques qui ne peuvent guère en contrôler les contenus (cf. à cet effet Gueorguieva 2008 ; Vaccari 2008 ; Yanoshevsky 2009) ${ }^{7}$. Je voudrais cependant poser ici la question autrement. Etant donné que ce sont les sites publics qui constituent l'objet de cet article, il ne s'agira pas de voir comment l'homme politique fait face aux messages des citoyens hostiles ou des supporters, mais de se demander comment les internautes se confrontent à la liberté que prend l'homme politique d'exploiter à son avantage les réseaux publics. On part donc de la prémisse que le site public appartient en premier lieu à l'internaute, et que l'homme politique n'y intervient qu'en second.

Dans cette perspective, on constate que les internautes récupèrent leur espace de libre expression en refusant de jouer le jeu proposé par l'homme politique et en détournant à leur profit les règles du jeu des réseaux sociaux. L'analyse de diverses ripostes des 
internautes montre que les réactions des cybercitoyens aux messages postés sont variées et vont d'une interprétation au pied de la lettre, en passant par une expression de soutien, à l'ironie et à l'appropriation des propos de l'homme politique.

\subsection{Formules d'adresse et soutien}

Même si l'usage mercantile que fait l'homme politique de Facebook est de fidéliser les cybernautes en manifestant son engagement à l'égard de ses interlocuteurs sur le Web, une partie des cybernautes prennent les enjeux politiques affichés pour un véritable programme politique, comme dans les posts suivants, qui réagissent au message de Ségolène Royal sur les mesures à prendre contre la sécheresse dans sa région :

(1) Truong Xuan Huynh

Bonjour, Madame Ségolène Royal,

j'ai entendu de la Sécheresse qui sévit la Région Poitou-Charentes depuis 10 jours,donc ,Il faut des mesures urgentes:limitations d'emploi d'eau ,surtout irrigations intensives ,ainsi le Ministre d'Environnement ,Le Gouvernement doivent prendre ces Mesures urgentes :la canicule d'été a été un exemple catastrophique avec des Mesures en retard...

August 12 at $8: 11 \mathrm{am}^{*}$ Report

(2) Brochard

JE la crois sincères (sic) dans sa démarche après tous elle pourrait toute envoyez baladé (sic) et joué les via pi a la télé non elle croit en ces idées et se bagarre pour nous pour se pays de toute façon contre Sarkosy (sic) il ni a qu'elle ou un autre mandat de sarkosy (sic) certainement sa collègue

August 18 at $1: 20 \mathrm{pm}$

(Je souligne)

Le message de Truong Xuan Huynh (1) affiche une réaction directe approuvant en fait et répétant la proposition de Royal sur Facebook. Ce message peut être donc perçu comme un signe de soutien, en même temps qu'un acte de responsabilisation, d'encouragement et de mobilisation: car ici Huynh presse sa représentante, en l'occurrence Royal, d'agir et de faire pression sur le gouvernement, et il fait en même temps des propositions précises d'action. Il s'agit bien d'une reconnaissance de l'idée de démocratie représentative (ou l'on délègue à son représentant le droit et l'obligation d'agir en son nom).

Or, le message (2), posté par Brochard, représente une manière toute différente d'aborder le post du représentant politique. Alors que Huynh réagit directement au sujet abordé par la représentante (à savoir, la sécheresse), Brochard se saisit de l'occasion pour manifester son soutien à Royal, face à l'actuel Président de la République. La réaction au post de la représentante de Poitou-Charentes n'est pour lui que l'occasion d'afficher son mécontentement à l'égard du gouvernement actuel et d'exprimer le désir que Royal gagne les élections dans l'avenir.

Mais la comparaison des deux messages est révélatrice aussi d'autres aspects. Par exemple, au niveau linguistique et pragmatique (l'usage des formules d'adresse et des pronoms personnels) : le message (1) de Huynh affiche une acceptation des règles du jeu imposées par la candidate, à savoir, l'adresse directe (« Bonjour, Madame Ségolène Royal »), mais correspond aussi aux formules de politesse conventionnelles dans le monde dit « réel ». Brochard (message 2), quant à lui, emploie un registre familier qui reflète non seulement le niveau de sa scolarité («via pi » pour VIP...), mais montre comment il perçoit le jeu communicationnel entre une personne politique et un 
sympathisant sur Internet - un échange à bâtons rompus qui n'engage pas forcément l'usage des formules qui signalent la hiérarchie dans l'adresse à un supérieur. Il semble croire verbatim aux suggestions de Royal en la soutenant à fond de façon naïve. Mais sa formulation à la troisième personne ("Je la crois sincères" [sic.]) démasque la simulation de la proximité : même si la personne politique s'adresse directement à l'internaute, l'usage de la troisième personne révèle qu'il ne s'agit pas ici d'une véritable interaction en face-à-face mais d'une communication en temps différé (asynchrone) dont le véritable dialogue est absent.

\subsection{Appropriation}

Parmi les voies d'émancipation des contraintes imposées par l'homme politique lorsqu'il s'empare de Facebook figure aussi l'appropriation. On définira comme appropriation toute modification du contrat de communication effectuée par l'internaute, qui change de registre (du formel au familier, par exemple) ou qui aborde des thèmes autres que ceux proposés par les représentants. Ainsi, dans l'exemple suivant, la réaction à l'adresse directe, mais formelle, de Royal qui invite ses supporters à participer à la fête de la fraternité, est suivie d'un changement de registre effectué par l'internaute : du formel-cordial (de l'adresse de la présidente de Poitou-Charentes) à l'adresse familière :

Marianne Gindrey

Ségolène tu es géniale ! Continue comme ça c'est bien !

Sun at $5: 21 \mathrm{pm} *$ Report

La manière dont l'internaute ci-dessus étend les limites de la cordialité exprimée par Royal sur le site contribue à l'illusion d'amitié que procure Facebook (Stein 2007). Là où les hommes politiques prétendent annuler la distance verticale (la hiérarchie), les internautes proposent d'éliminer la distance horizontale en feignant des rapports amicaux. Par exemple, cette même internaute écrit par ailleurs à Royal pour la prévenir de son absence à la fête annoncée, mais lui parle aussi de ses hésitations professionnelles :

Marianne

Discussion board, on August 13, 2009 at $10: 36$ am

Ségolita,

Je ne pourrai pas aller à la fête de la fraternité car Montpellier et trop loin de chez moi. Je suivrai cette fête magnifique sur internet. Je pense à mes études et plus tard j'aimerai faire maquilleuse de mannequins. Je t'encourage pour le Zénith. Bises de Marianne Gindrey

Le recours aux procédés de proximité : l'usage du prénom, d'un diminutif (« Ségolita »), le tutoiement et l'information privée communiquée par Marianne Gindrey à Royal laissent entendre un rapport de familiarité entre les deux femmes, même si ce rapport n'existe pas en dehors de l'espace virtuel. L'appropriation dans ce cas consiste dans le passage d'un message standard d'encouragement à une information qui ne concerne que l'internaute spécifique, c'est-à-dire son choix de carrière. En réagissant de la sorte, l'internaute ramène Royal sur le terrain de Facebook qui vise à multiplier les " amis ", alors que la représentante utilise ce dispositif comme moyen d'information et d'embauche politique de soutien. Par ailleurs, cet exemple semble confirmer la règle d'Internet qui consiste en la fragmentation des intérêts (Dahlgren 2005), phénomène qui s'affirme aussi dans le message suivant, où la femme de l'immigré Nazam Javid 
s'adresse directement à « Nicolas ", dans l'espoir qu'il interviendra pour que son mari puisse travailler légalement :

Nazam Javid

Bonjour nicolas, je suis le femme de nazam et je voulais juste vous demander comment faire pour que mon mari et moi on puissent vivre tranquillement sans avoir de probleme de papier. Nous nous fréquentons depuis 2 ans et après nous etres marié, on s'apperçois qu'on nous refuse le droit du travail (enfin a mon mari).

[...] Pouvez vous svp nous répondre. Merci... Anne Ferrarini (sic)

August 10 at $2: 05 \mathrm{am}^{*}$ Report

Alors que l'homme politique use des procédés de proximité, mais n'offre en aucun cas un soutien direct à qui que ce soit, l'internaute demande ici une aide personnalisée. Cette demande directe d'aide reflète soit une croyance en la bonne volonté de l'homme politique, soit une manière de lui rappeler ses promesses en lui demandant d'agir spécifiquement. Un cas extrême consiste à se saisir de l'espace prévu par l'homme politique pour un usage privé doté d'un enjeu particulier. Ainsi, l'internaute Mohammed Chefcahouni utilise le cadre du message suivant de Sarkozy (janvier 23 2010):

Nicolas Sarkozy Je m'exprimerai à la télévision lundi, pour répondre aux questions des Français et parler des grands enjeux de l'année 2010. N'hésitez pas à me faire part de vos interrogations, et des sujets qu'il vous paraît important que j'aborde. Merci pour votre soutien en cette année où nous allons travailler dur pour servir l'intérêt de la France

- afin d'afficher une annonce immobilière :

January 23 at 12

Mohammed Chefchaouni

je vend un terrain de $5000 \mathrm{~m}$ sur la route de Meknès,400m bâtis,château d'eau,fausse septique,un puits+elect

tel : $33+212+0668841239$

Dom :33+212+0535749563

e-mail :chefchaouni_m@hotmail.com

May 27 at $7: 31 \mathrm{pm}^{*}$ Flag

L'œil perspicace du lecteur apercevra sans doute que la date du post de l'internaute est nettement plus tardive : l'internaute se sert du site du Président de la République pour promouvoir ses propres affaires. Un autre exemple consiste en une tentative de diffuser un message politique en accaparant l'espace du représentant. Ainsi Cada Wan inonde tous les messages du Président, du 11 avril 2010 au 9 mai 2010, avec un post prônant l'intervention de Sarkozy dans l'affaire récente de la flotille en partance pour Gaza.

Les internautes se servent de la page officielle sur Facebook comme plateforme pour diffuser leurs messages afin de contacter et de mobiliser d'autres internautes. On constate alors que le changement de propos ainsi que l'usage extrême que font les internautes du principe du temps différé sont des stratégies destinées à regagner le contrôle du dispositif public.

\subsection{Ironie et injures}

Une autre manière de rompre avec les contraintes imposées par l'homme politique lorsqu'il exploite Facebook, consiste en l'usage de l'ironie. Par exemple, aux vœux des bonnes vacances d'été de Royal, le Clebs répond: 
C'est cool! elle proférera moins de conneries en prenant la distance estivale : ça nous fera des vacances.

July 24 at $9: 55$ am * Report site public: les vœux et la bonne volonté de l'homme politique sont confrontés à des " tomates virtuelles » : au message précédent de Sarkozy (23 janvier 2010) où il annonce une prochaine intervention à la télévision et encourage les internautes à lui faire part des leurs interrogations en les remerciant de leur soutien «en cette année où nous allons travailler dur pour servir l'intérêt de la France », Gérard Pompée répond (message du 26 mai 2010) : "Bonjour, vous ètes un menteur car en 2007 vous avez promis de ne pas touché la retraites a 60 ans (sic)».

41 Le fait que les posts ironiques et les injures, c'est-à-dire des messages d'opposants, soient laissés en ligne, sans retouche aucune, atteste que les personnes politiques renoncent à l'idée de pouvoir contrôler les messages des internautes. Le fait même de laisser parler les gens en toute liberté, sans entraver leur parole, aussi négative soitelle, produit une image plutôt favorable de l'homme politique ${ }^{8}$.

\subsection{La part des débats}

Organisés par la personne politique ou spontanés, des débats argumentés ont lieu sur le site de Ségolène Royal et, plus rarement, sur celui de Sarkozy. Il est intéressant de noter en passant que ces débats prennent place surtout dans l'espace "Discussions " consacré à cet effet chez Royal (les discussions sont initiées par les internautes et non par Royal). Par exemple, le débat intitulé «Comment il faut aider la Grèce ? " crée en avril 232010 a suscité à ce jour 105 posts. En général, les personnes participants à ce débat adhèrent à l'étiquette $d u$ "monde réel " (formules de politesse, langue plus soutenue des lettres), et semblent utiliser leur véritable identité (avec en général leur propre photo). Les ripostes sont longues, comptant parfois 40 (!) lignes, développent plusieurs arguments et répondent souvent explicitement aux arguments présentés par d'autres interlocuteurs sur le site (on s'adresse directement à celui à qui on répond: "Cher Panayotis ", etc.).

Quoique moins élaborés, reflétant un niveau moindre de scolarité et obéissant plutôt à la Netiquette (brièveté et laconisme du message, usage d'un pseudonyme, etc.), les débats peuvent néanmoins avoir lieu aussi sur les "murs " des deux personnes politiques. Par exemple, le post de Royal concernant l'affaire de la burqua et de l'attitude manifestée à cet égard par le gouvernement français, est suivi des réactions argumentées suivantes (je ne reproduis ici qu'une partie des ripostes et j'omets pour des raisons de brièveté les injures, les méta-remarques, et les ripostes générales de soutien ou d'opposition) :

Ségolène Royal C'est trop, beaucoup trop ce tapage électoraliste sur la burqua, ces mises en cause qui stigmatisent toute une communauté sans même régler correctement la question. J'invite Nicolas Sarkozy à concentrer son effort sur les 
vrais problèmes et...

April 27 at $11: 09 \mathrm{pm} *$ Comment

Jose Marin

il faut l'interdire point barre ! ça stoppera ce tapage et ce sera clair , on ne peut pas accepter tranquillement ce signe mortifere pour les femmes afghanes et des regimes « islamiques »!

attention si la gauche ne comprend que les français de toutes confessions et athées ne veulent pas de ces fantomes, négations des femmes, et si elle continue a tergiverser, elle en subira les retombés electorale !

April 28 at $3: 20 \mathrm{pm} *$ Flag

Maxime Jousset

je suis tout à fait d'accord avec vous mais la droite et l'extrème et la gauche ps VALS en font leurs chemin de bataile

les Français écoute comme il le disent si bien on entend que ceci, alors comment faire pour ne pas en parler?

April 28 at $3: 27 \mathrm{pm}$

Patrick Choquez

Pas d'accord avec vous, bien qu'étant socialiste. La burqua : rien de plus dégradant pour une femme. La sollution est simle, interdire purement et simplement quelconque signe religieux dans les lieux publics, même traitement pour les catholiques, les boudhistes, les musulmans et le juifs !

Chacun est libre de sa pensée mais il le garde pour soi, la pratique chez soi ou dans les lieux de cultes.

Arrêtons de nous emmerder avec ces histoires de religions responsables de trop nombreuses victimes à travers le monde !

April 29 at $12: 19 \mathrm{am}^{*}$ Flag

Norbert Van der Meulen

n'empêche que le PS ne doit pas éluder le sujet encore une fois,pas de dérogation à la laïcité, sans stigmatiser personne.La Laïcité est la seule voie à l'émancipation des femmes, et de l'égalité entre les hommes et les femmes.

salut et fraternité, Norbert

May 8 at $3: 51$ am * Flag

Quoique le flaming, les déclarations ou le echoing (approbation, répétition en riposte des propos énoncés dans le post de la personne politique, sans argumentation) dominent le "mur», on trouve des discussions entre deux ou plusieurs participants, en temps différé. Il faut noter que souvent il revient aux mêmes internautes de se prononcer sur une question, et que ce sont souvent les mêmes qui réagissent de façon plus ou moins argumentée ou plus ou moins « enflammée ». Il en résulte que ce n'est pas le dispositif technologique qui détermine ici la possibilité ou non d'une argumentation, ni l'usage qu'en en fait la personne politique (selon qu'elle crée ou non sur son site une catégorie spécialisée pour les discussions). C'est plutôt le style personnel de l'internaute (qui est libre de choisir le canal et le mode d'expression [registre, propos, etc.] sans intervention ou restriction de la part de la personne politique à qui appartient le site) qui décide s'il y aura ou non débat. Il s'avère alors que l'idéal de la sphère publique (échange rationnel des arguments) peut se réaliser aussi sur des réseaux sociaux comme Facebook, et n'est pas uniquement affaire de flaming.

\section{En guise de conclusion}

Sans doute peut-on trouver sur Facebook des débats qui seraient selon la sociologie électorale un indicateur parmi d'autres de participation politique (Mayer \& Perrineau 1992, cité chez Greffet \& Wojcik 2008 : 22). Mais ces débats ne constituent pas l'essentiel 
des échanges sur ce dispositif public. Qui plus est, ils ne sont pas forcément organisés par l'homme politique (contrairement aux débats de démocratie participative/ délibérative organisés par les autorités), sauf si l'on considère que l'homme politique en est responsable dans la mesure où il a ouvert un espace propice au débat (à savoir le marqueur "Discussions »). Souvent, les débats excèdent les propos proposés par les hommes politiques. Facebook peut servir de cadre à des discours dotés d'enjeux politiques (Greffet \& Wojcik 2009: 23-24), mais c'est aux internautes de choisir de coopérer ou non. On trouve alors sur Facebook les tendances identifiées sur d'autres modes d'échanges électroniques (Michaël Dumoulin cité chez Greffet \& Wojcik 2008 : 25 et par Flichy $2008: 163)$ : des monologues interactifs ou chaque participant réitère son propre point de vue et rejette ou critique systématiquement les propos des autres intervenants au lieu de solliciter des opinions, des éclaircissement ou des justifications de la part des internautes - mais aussi des demandes à s'engager dans de véritables discussions. Ce que l'analyse des modalités d'échange entre l'homme politique et les internautes sur Facebook rajoute à l'approche de la délibération (Greffet \& Wojcik 2008: 27), c'est qu'au-delà de l'idéal habermassien qui fait défaut sur les réseaux sociaux, comme le montrent Greffet et Wojcik, il y a différentes manières pour les internautes de se faire entendre, d'assigner des limites à l'homme politique, de contrôler ce qu'il fait. Ceci confirme alors l'indépendance de l'internaute à l'égard des dirigeants.

\section{BIBLIOGRAPHIE}

Site officiel de Ségolène Royal, Désirs d'avenir, http://www.desirsdavenir.org/

Site officiel de Nicolas Sarkozy pendant les élections, Ensemble tout devient possible, http:// www.sarkozy.fr/home/

Site officiel de Ségolène Royal sur Facebook, http://www.Facebook.com/home.php?\#/ segoleneroyal?ref=ts

Site officiel de Nicolas Sarkozy à l'Elysée, http://www.elysee.fr/accueil/

Site officiel de Nicolas Sarkozy sur Facebook, http://www.Facebook.com/search/? q=nicolas+sarkozy\&init=quick\#/nicolassarkozy?ref=search\&sid=610427994.700881947..1

Anstead, Nick \& Andrew Chadwick, 2008. « Parties, election campaigning, and the Internet Toward a comparative institutional approach ", Chadwick, Andrew \& Philip N. Howard (éds). The Routledge Handbook of Internet Politics (New York and London : Routledge), 56-71

Barthes, Roland. 1957. Mythologies (Paris : Seuil)

Bonnafous Simone et al. (éds). (Groupe Saint-Cloud). 1999. L'image candidate à l'élection présidentielle de 1995. Analyse de discours dans les médias (Paris : L’Harmattan)

Cardon, Dominique, 2009. « Présentation », Réseaux 2 : 154, 9-12 
Clark, Amanda. 2010. « Les médias sociaux. 4. Utilisations politiques et conséquences pour la démocratie représentative », Bibliothèque du parlement, étude générale, Publication n. 2010-10-F, 22 Mars. 1-10

Cong Huyen $\mathrm{Nu}$, Phung Tien. 1999. «Les clips de J. Chirac : de l'homme ordinaire (1988) au symbole présidentiel (1995)»; Bonnafous, Simone et al. (éds) (Groupe Saint-Cloud), L'image candidate à l'élection présidentielle de 1995. Analyse de discours dans les médias (Paris : L'Harmattan), 49-61

Dahlgren, Peter. 2005. « The Internet, Public Spheres, and Political Communication: Dispersion and Deliberation », Political Communication, $22: 147-162$

Dalsgaard, Steffen. 2008. « Facework on Facebook The presentation of self in virtual life and its role in the US elections », Anthropology Today 24:6, 8-12

Fairclough, Norman. 1992. Discourse and Social Change (Cambridge: Polity Press)

Fairclough, Norman \& Anna Mauranen. 1997. « The conversationlization of political discourse. A comparative view », Political Linguistics, Belgian Journal of Linguistics 11, 89-116

Flichy, Patrice. 2008. «Internet et le débat démocratique », Réseaux $26: 150,160-185$

Goupil, Sylvie. 2007. « Gouvernement en ligne et démocratie : vers une mutation de la relation citoyenne à l'Etat?», Politique et Sociétés $26: 1,27-46$

Greffet, Fabienne \& Stéphanie Wojcik « Présentation », Réseaux 26 : 150, 9-16

Gueorguieva, Vassia. 2008. « Voters, MySpace, and YouTube : The Impact of Alternative Communication Channels on the 2006 Election Cycle and Beyond ", Social Science Computer Review $26,288-300$

Habermas, Jürgen, 1978 [1963]. L'espace public. Archéologie de la publicité comme dimension constitutive de la société bourgeoise (Paris : Payot)

Horton, D. \& Whol, R.R. (1956). « Mass Communication and Parasocial Interaction : Observation on Intimacy at a Distance », Psychiatry $19: 3,215-229$

Katz, Elihu. 1996. «And Deliver us From Segmentation », Annals of the American Academy of Political and Social Science 546, 22-33

Le Bart, Christian. 2009. « Les présidentiables de 2007 entre proximité et surplomb. Nicolas Sarkozy et Ségolène Royal vus par Libération », Mots 89, 39-55

Ledun, Marin. 2005. La démocratie assistée par ordinateur : Du sujet politique au consommateur à caractère politique (Paris : Connaissances et Savoirs)

Oberdorff, Henri. 2009. La démocratie à l'ère numérique (Grenoble : Presses universitaires de Grenoble)

Poster, Mark. 1997. « Cyberdemocracy : The Internet and the Public Sphere », Porter, David (éd). Internet Culture (New York and London : Routledge), 201-218

Rousseau, Jean-Jacques. 2008 [1762]. Du contrat social ou Principes du droit politique, Soares, Sálvio M. (éd), MetaLibri [version en ligne]

Small, Tamara A., 2008. « The Facebook Effect ? Online Campaigning in the 2008 Canadian and US Elections ", Policy Options, Novembre, 285-287

Stein, Joel. 2007. « Friends in High Places », Time Magazine 11 juin, 64 
Tolson, Andrew. 2001. « 'Being Yourself' : The Pursuit of Authentic Celebrity », Discourse Studies $3: 4,443-457$

Vaccari, Christian. 2008. «Surfing the Elysée : The Internet in the 2007 Elections », French Politics $6,1-22$

Vedel, Thierry. 2003. « Political Communication in the Age of the Internet », Maarek, Philippe J. \& Gad Wolfsfeld (éds). Political Communication in a New Era (London, USA, Canada : Routledge), 41-59

Vedel, Thierry. 2000. «L'internet et la démocratie », Les cahiers français 295, 25-30

Vanbremeersch, Nicolas. 2009. De la démocratie numérique (Paris : Seuil)

Westling, Mike. 2007. « Expanding the Public Sphere: The Impact of Facebook on Political Comunication », Thenewvernacular.com (May) [En ligne : http://www.thenewvernacular.com/ projects/Facebook_and_political_communication.pdf]

Williams, Christine B. \& Girish J. “Jeff” Gulati. 2007. « Social Networks in Political Campaigns : Facebook and the 2006 Midterm Elections », Paper delivered at the 2007 Annual Meeting of the American Political Science Association, Chicago, Illinois, August-September 2007

Yanoshevsky, Galia. 2009. «L'usage des vidéo-blogs dans la campagne présidentielle de 2007 : Vers une image plurigérée des candidats ", Mots 89, 57-68

Yildiz, Hélène, 2002, « Internet : un nouvel outil de communication multidimensionnel », Serfaty, Viviane (éd). L'Internet en Politique, des Etats-Unis à l'Europe (Strasbourg : Presses Universitaires de Strasbourg), 275-290

\section{NOTES}

1. Je l'entends ici dans le sens qui lui est attribué par Fairclough (1992) à savoir, que la communication publique ressemble de plus en plus aux conversations ordinaires.

2. Ibid., cf. http://www.facebook.com/facebook.

3. On sait qu'initialement, Facebook était un outil destiné aux étudiants des collèges américains. Sur ses modalités de fonctionnement à l'origine, et sa politisation à partir de 2006, on consultera Williams \& Gulati 2007 et Small 2008.

4. Aux élections canadiennes, par exemple, Small (2008) constate que les profils de Facebook étaient assez statiques et comportaient des photos de la campagne et des pubs télévisées des partis politiques.

5. Pour en apprendre plus, cf. Yanoshevsky 2009.

6. Sans doute obéit-il au principe de If you can't beat them, joint them : car la presse échotière ainsi que les hebdomadaires d'orientation politique comme L'Express et Le Nouvel Observateur préconise déjà bel et bien le mélange du privé et du public. Cf. les photos de Sarkozy et de Ségolène Royal en maillot de bain pendant la campagne présidentielle.

7. Bien que la question du contrôle de l'image ait toujours été un enjeu important, dans les anciens medias également : « [1]e positionnement par rapport à l'image télévisuelle et la tentative de la maîtriser [étaient] à la base des choix stratégiques de tout candidat » (Bonnafous \& al. 1999).

8. On se souviendra du site official de Hillary Clinton pendant les présidentielles aux Etats-Unis en 2008. Des opposants se sont plaints de l'incompétence de Clinton, car pendant des semaines, un vidéo-clip hostile qu'ils ont téléchargé sur son site et qui la représentait en guignol sorcière y a figuré sans que personne n'ait veillé à l'enlever. 


\section{RÉSUMÉS}

A travers l'analyse des interactions entre hommes politiques et internautes sur Facebook après les élections présidentielles de 2007 en France, cet article vise à montrer comment les réseaux sociaux sont exploités par les deux parties. Au départ un réseau décentralisé qui lie les gens sans intervention politico-hiérarchique, Facebook est mobilisé par les hommes politiques au service de leur besoins. Quelles en sont les conséquences pour la sphère publique : s'agit-il d'un outil qui permet à l'internaute de déjouer les usages de marketing politique qu'en fait l'homme politique ?

The purpose of this article is to show how social networks, and more specifically Facebook, are used by politicians and their supporters. This is achieved through a detailed analysis of exchange of posts between politicians and surfers in the period following the presidential election campaigns in France of 2007. It is shown how politicians take advantage of Facebook, despite its initially being a decentralized social network, connecting people without a political or hierarchic purpose. The article attempts to show some of the consequences of such an exploitation of a public network for the public sphere and asks how much leverage there is for the surfers/citizens in moderating political marketing on Facebook.

\section{INDEX}

Keywords : Facebook, presidential election campaign, proximity, Web 2.0

Mots-clés : campagne présidentielle, Facebook, proximité, Web 2.0

\section{AUTEUR}

\section{GALIA YANOSHEVSKY}

Université Bar Ilan, ADARR 